\title{
Substrate Influence on the Recrystallization of 1,2-Dodecanediol: An Atomic Force Microscopy Study
}

\author{
S. K. Dora \\ NanoMagnetics Instruments Pvt. Ltd., Turkey
}

Copyright $\bigcirc 2017$ by authors, all rights reserved. Authors agree that this article remains permanently open access under the terms of the Creative Commons Attribution License 4.0 International License

\begin{abstract}
1,2-dodecanediol otherwise known as lauryl glycol (LG), a skin conditioning agent in many skin cosmetics was recrystallized from chloroform on to a number of different technical substrates to understand how material properties affect their recrystallization behavior at the molecular level. For this purpose, four different technical substrates were chosen based on their polarity and crystallinity. On highly oriented pyrolytic graphite or HOPG (nonpolar, crystalline), LG recrystallization formed parallelogram-like-structures in the initial stage. On nonpolar, amorphous substrate like glassy carbon (GC) stalactite-like-structures were observed. Contrary to this, on polar substrates like mica (polar, crystalline) and glass (polar, amorphous), LG molecules were recrystallized as thin film. The morphological changes of LG at different time intervals were investigated and their mechanism of recrystallization on all the substrates is discussed in detail for the first time. Direct view of the recrystallization process at the molecular level on different technical substrates can be used as a model in understanding how these molecules as a constituent in skin cosmetic products behave, when applied to skins of varying physical and chemical barrier ultimately helping in better cosmetic development.
\end{abstract}

Keywords 1,2-dodecanediol, Tapping Mode AFM, Recrystallization, Substrate Effect

\section{Introduction}

Straight-chain 1,2-alkanediols are multifunctional ingredients with moisturizing and antimicrobial activities [1-7]. They have been used in household and cosmetic products since very long time as solvents [1], viscosity modifiers [1, 2], cryoprotectors [3] as well as moisturizers [4]. Due to their low toxicity and strong antimicrobial properties, they have replaced the traditional preservatives such as parabens, potassium sorbate, sodium benzoate, and formaldehyde-releasing compounds in cosmetic products [1,
5]. 1,2-dodecanediol otherwise known as Lauryl glycol (LG) is one such alkane diol having a 12-carbon alkane chain with $-\mathrm{OH}$ groups at 1,2 position. It has a variety of potential uses. For example, it is used as a skin conditioning agent in many skin cosmetic products [6]. Wilbur Johnson Jr and coworkers demonstrated the dermal (skin) absorption rate was up to $40 \%$ for LG [6] on pig skin. Other potential applications reported in literature are as follows. It has been used to prepare lipophilic diamines and amino alcohols [7] which show activity against tuberculosis bacteria. LG found application as a reducing agent in the preparation of $\mathrm{Fe}(\mathrm{II})$, $\mathrm{Co}(\mathrm{II})$ and $\mathrm{Ni}$ (II) nanoparticles [8]. Freitas et al. have demonstrated the importance of LG in the Ketalisation of ethyl levulinate resulting in a new class of biodegradable surfactants [9]. The use of LG as a constituent in skin cosmetics is the main driving force for the work presented here. The obvious question comes to mind why some skincare preparations are effective and others are not? To understand this concept one needs to look at the absorption behavior of various constituents of these products at the molecular level. In this aspect LG as one of the constituent was recrystallized on different technical substrates to understand their recrystallization mechanism in detail. Atomic force microscopy (AFM) in tapping mode was employed for this purpose. AFM is a surface technique used to characterize topography at extremely high resolution. A sharp probe is brought into close proximity with the sample to be analyzed. Probe and sample are then moved relative to each other in a raster pattern, and a quantity is measured in a serial fashion at discreet locations (pixels). AFM operates in either contact mode or tapping mode. The tapping mode was developed to minimize the lateral force during scanning and is the most dominant mode in AFM field at this moment routinely allowing high resolution topographic imaging of sample surface that are easily damaged, loosely hold to the substrate or difficult to image by contact mode. In this mode, the cantilever is oscillated at or near its resonance frequency. As the cantilever approaches the sample surface, depending on the interaction between sample and cantilever, the cantilever undergoes a change in amplitude and frequency. 
This change in amplitude is measured by a feedback control and plotted as an AFM image. Details of tapping mode AFM operation can be found in the literature [10]. In the last few decades, AFM played a vital role to study absorption and recrystallization mechanisms at the nano level [11-15]. The advantage of understanding the detailed absorption mechanism of LG at the nanoscale is actually two-fold. First, it will help understanding how material properties affect its absorption behavior. For instance, understanding of absorption of LG molecules on non-polar substrate like HOPG may be used as a model to understand their absorption on human skin which is also nonpolar in nature. Second, it will develop interest in scientists especially those working in the field of cosmetics to use AFM as an effective tool to learn about the absorption of other diols and constituents used in cosmetics at the nanoscale. Understanding the detailed absorption mechanism of such constituents will ultimately help the scientists to design better cosmetic products. For this purpose, in this study a low concentration of $0.4 \mathrm{mg} / \mathrm{mL}$ was used to understand their absorption phenomena at the LG-substrate interface on a number of different technical substrates. The technical substrates were chosen based on their polarity and crystallinity. They are highly oriented pyrolytic graphite in short HOPG (polar, crystalline), glassy carbon in short GC (nonpolar, amorphous), glass (polar, amorphous) and mica (polar, crystalline). Understanding the phenomenon at the nano level is also beneficial from consumer point of view as recent advances in nanotechnology has made it possible to manufacture products that provide dramatic visible improvements in skin quality in a matter of few weeks or in some cases in few days.

\section{Experimental}

In this study four different types of substrates are used depending upon their polarity and crystallinity. They are classified as HOPG (nonpolar, crystalline), glassy carbon (nonpolar, amorphous), mica (polar, crystalline) and glass (polar, amorphous). For HOPG and mica, new substrate surface was prepared by removing few top layers using adhesive tape. Cleaning of microscopic glass slides were carried out by putting them in a beaker containing ethanol under ultrasonic bath for 15 minutes. Sonication is a technique where sound energies with frequencies $>20 \mathrm{kHz}$ are applied to the samples to agitate particles for various purposes. In this experiment sonication was used to remove any adhering dust particles to substrates used in the experiment. Commercially available glassy carbon (GC) from HTW Germany was purchased and was cleaned like glass before using in this study.

AFM measurements were carried out in tapping mode using an Agilent 5500 system under ambient condition. PPP-NCH cantilevers from Nanosensors with a nominal resonant frequency of $\sim 300 \mathrm{kHz}$ were used for observation of crystal growth process at different time intervals. Imaging conditions employed were a scan size of $4.9 \times 4.9 \mu \mathrm{m}^{2}$, a scan rate of $0.612 \mathrm{~Hz}$, and an image size of $256 \times 256$ pixels. The set point was chosen close to the upper limit possible and all measurements were performed at room temperature $\left(\sim 22-24^{0} \mathrm{C}\right)$. Recrystallization process at different time intervals was observed by AFM, after applying a $10 \mu \mathrm{L}$ droplet of $0.4 \mathrm{mg} / \mathrm{mL} \mathrm{LG}$ mass in chloroform on to different substrates. The chloroform took ca. 30 seconds to evaporate from the surface leaving the LG molecules attached to the substrates. The substrates were then mounted to the AFM sample plate by using metal clips separately. The first image acquisition started as soon as possible after the chloroform evaporation, typically after $\sim 10$ minute. AFM images were taken from the same substrate area at different time intervals applying a constant scan rate of $0.612 \mathrm{~Hz}$. Experimental data was analyzed by using the program WSxM (Version 4.0 Beta 8.3; Nanotec Electronica, Madrid, Spain) [16].

\section{Results}

This section describes the details of morphological changes of LG molecules at various time intervals after recrystallizing them on different technical substrates. Depending on the nature of substrates used, the recrystallized LG structures varied. The principle findings of these structural variations with respect to time are presented here.

\subsection{LG Recrystallization on HOPG}

A series of AFM images at different time intervals demonstrating the morphological changes after recrystallizing $0.4 \mathrm{mg} / \mathrm{mL} \mathrm{LG}$ molecules from chloroform on to a freshly cleaved HOPG surface is presented in figure 1. Structures which look like parallelograms, from here onwards will be called as parallelogram-like-structures appeared on the HOPG surface in the initial stages of recrystallization (see fig. 1a, time $\mathrm{t} \sim 13 \mathrm{~min}$ ). By imaging different areas on HOPG after freshly recrystallizing LG in a number of independent experiments it is found that the lengths, widths and heights of these structures vary randomly at different places on HOPG surface. The terms length, width and height of these structures are defined in the schematic (see fig. 1g). The average width of these structures varies between $\sim 100 \mathrm{~nm}$ to $\sim 400 \mathrm{~nm}$. Width profile of two such structures (see fig. 1e and 1f) marked by white and black line in fig. 1a gives values of $\sim 270 \mathrm{~nm}$ and $\sim 360 \mathrm{~nm}$ respectively. The random variation of widths within $\sim 100-400 \mathrm{~nm}$ range makes it practically impossible to draw any meaningful statistics for their width variation. Their length varies from few hundred nanometers to several microns. For example, the shortest (white arrow mark) and longest structure (black arrow mark) in fig. 1a have lengths of $\sim 200 \mathrm{~nm}$ and $\sim 2.5 \mu \mathrm{m}$ respectively. Structures of longer length were also observed in other areas of HOPG surface (figure not shown) indicating that their lengths also vary randomly. The maximum height 
of these structures was found to be $\sim 24 \mathrm{~nm}$. As examples the height profiles along black and white line in fig. 1a (see profile heights in fig. 1e and 1f) have heights of $\sim 24 \mathrm{~nm}$ for both the structures. This holds true for other areas of the HOPG surface also. Just to emphasize that all heights are measured from the lowest point on the surface assuming it to be HOPG surface. A closer look also revealed that individual structures are having multiple layers. The height profile of
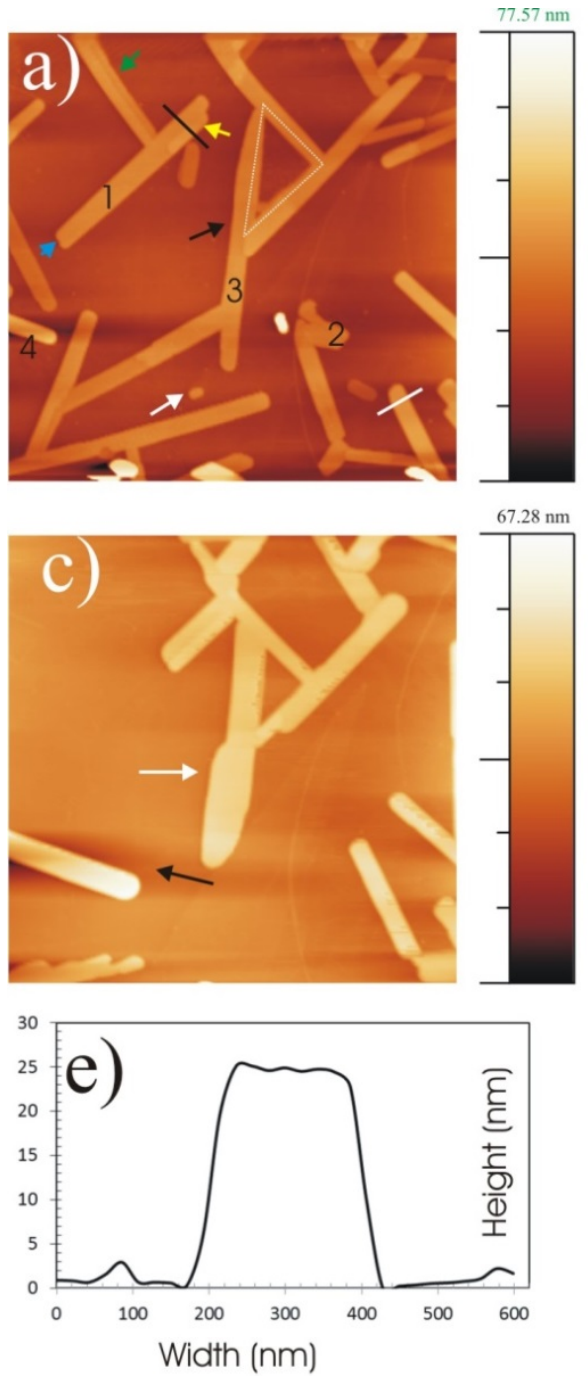

one such visible layer has given a height of $\sim 3.1 \mathrm{~nm}$ (see the yellow arrow in fig. 1a and the vertical red line in fig. 1f). Some of these parallelogram-like-structures also seem to be partially overlapping sidewise (green arrow, fig. 1a) or merged completely in sidewise fashion along their width to form structures of larger width (blue arrow, fig. 1a). Further, they are also found to be arranged in a triangular fashion on HOPG (dotted white triangle marked in fig. 1a).
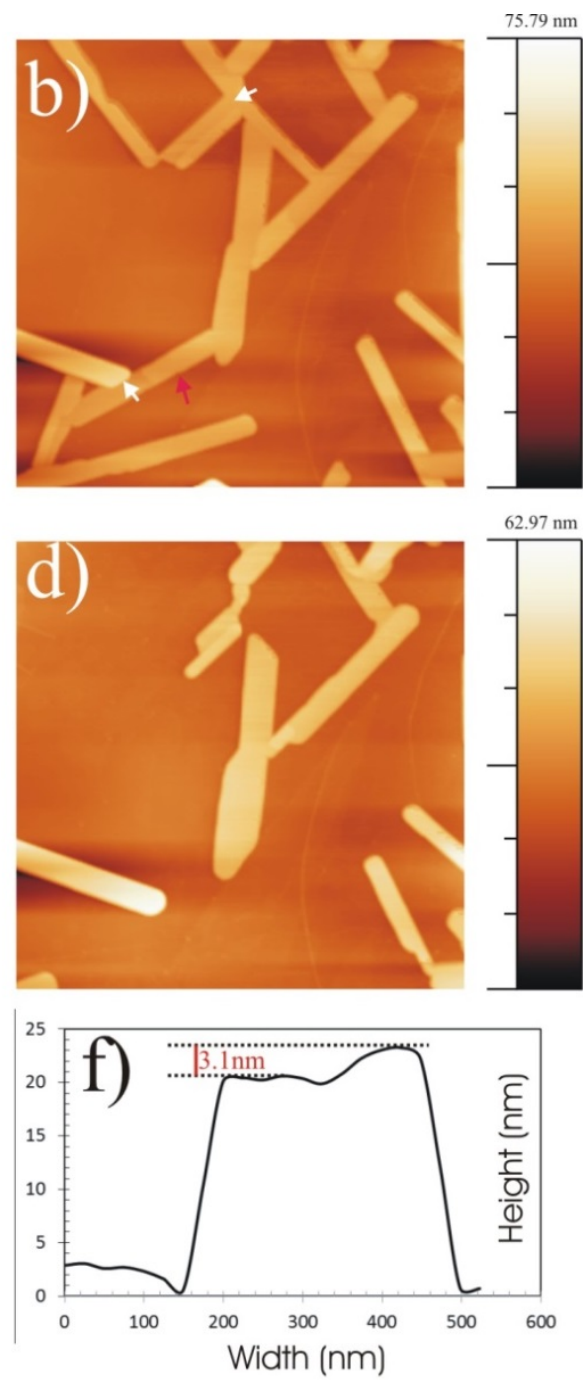

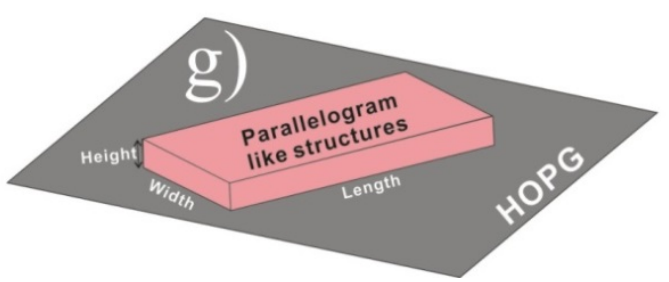

Figure 1. AFM images at different time intervals showing the morphological changes of LG crystals after recrystallizing $0.4 \mathrm{mg} / \mathrm{mL}$ LG solution (chloroform solvent) on to HOPG substrate. Figure 1a taken after $\sim 13$ min shows parallelogram-like-structures of variable length and width arranged in triangular fashion. Figure 1b, c taken after $\sim 126 \mathrm{~min}$ and $\sim 257 \mathrm{~min}$ demonstrates the dissolution/growth of few parallelogram-like-structures (for details see text under "LG crystallization on HOPG"). Recrystallization reached a saturation point at $\sim 1067$ min (fig. 1d) and no further morphological changes are observed after that. Figure 1e, f shows profiles across white and black line in fig. 1a. Figure 1g shows the schematic used to describe length, width and height for parallelogram-like-structure. 
The observed structure in fig. 1a has undergone considerable morphological changes after $\sim 2$ hours (fig. 1b, $\sim 126 \mathrm{~min}$ ). Simultaneous dissolution and growth of few individual parallelogram-like-structures were noticed during image acquisition. A closer look also revealed that a single parallelogram-like-structure can both dissolve and grow simultaneously. For example, the one marked by number ' 1 ', in fig. 1a was found to be undergoing dissolution from one end and growth at the other end in fig. 1b. Complete dissolution of some of these structures is also observed (see the one marked by number ' 2 ' in fig. 1a disappeared completely in fig. 1b). Few of these structures were found to have grown both along their length and width but their heights remain constant. For instance structures in fig. 1a marked by number ' 3 ' has grown along its width and marked by number ' 4 ' has growth both along length and width in fig. 1b. In addition, growth of individual structures was found be terminating by the presence of obstacles e.g. another parallelogram-like-structure across its growth direction. See for instance, the white marked arrow junctions in fig. $1 \mathrm{~b}$ acted as terminating points for the growth of the parallelograms.

Further topographical changes of the same surface recorded after 4 hours $(\sim 257 \mathrm{~min})$ are shown in fig. 1c. Looking at the minute morphological change after a time interval of $>2$ hours between fig. $1 \mathrm{~b}$ and $1 \mathrm{c}$, it is safe to assume that the kinetics (details of kinetics for LG growth/dissolution will be published in a separate paper) of growth/dissolution has decreased with passage of time. However, the recrystallization process has not ceased completely at this stage as it is found that the width and length of some of these structures continue to increase (black and white arrow in fig. 1c) and other structures continue to dissolve (red arrow in fig. $1 \mathrm{~b}$ dissolved in fig. 1c). At this stage one of the parallelogram-like-structure whose growth was terminated (see lower left white arrow marked parallelogram in fig. 1b) due the presence of another one across it, started to grow again once the latter got dissolved completely. Even after more than 4 hours, no change in heights of the remaining structures is observed. At about 18 hours the growth/dissolution process has completely ceased indicating a saturation point has reached (fig. 1d, 1067 min). No further morphological changes were observed even if the experiments were carried out up to 21 hours (data not shown).

\subsection{LG Recrystallization on Glassy Carbon (GC)}

Recrystallization of LG on GC substrate resulted in the formation of stalactite-like-structures (see fig. 2a, time $\sim 13$ min). By scanning several different areas of GC in a number of different experiments after freshly recrystallizing LG on to them, it was also found that these stalactite-like-structures are distributed in an inhomogeneous manner on the GC surface. Each such stalactite-like-structure seems to be formed by the agglomeration of many individual structures which are overlapped partially on top of others to form the complete stalactites. A closer look at one such individual structure (see black arrow in fig. 2 a) revealed its similarity to the ones observed on HOPG with the exception that the edges do not look so sharp and distinct compared to those observed on HOPG. Variation of heights and widths of individual stalactites are random and it is practically impossible to make any meaningful statistics. However within the same stalactite, the widths and heights seem to decrease towards the pointed direction. For example, the heights and widths measured at dotted black and white lines in fig. 2a gives values of $\sim 70 \mathrm{~nm}, \sim 90 \mathrm{~nm}$ for heights and $\sim$ $430 \mathrm{~nm}, \sim 630 \mathrm{~nm}$ for widths respectively (see profiles in fig. $2 \mathrm{e}$ and $2 \mathrm{f}$ for black and white dotted line). Overlapping of stalactites is also observed and their overlapping seems to have certain directional in nature. As an example, the overlapping stalactites shown in fig. 2 a made angles of $30^{\circ}$ and $60^{\circ}$.

Simultaneous dissolution as well as growth was observed in certain locations of the stalactites with passage of time. This process is similar to those observed on HOPG substrate with the exception that dissolution/growth of stalactite-like-structures are observed here instead of individual parallelogram-like-structures on HOPG. Figure $2 \mathrm{~b}$ taken after $\sim 70$ min shows complete as well as partial dissolution of areas marked by blue and red arrow heads in fig. 2a respectively. A closer look also revealed the LG mass in direct contact with the substrate was dissolved preferentially at this stage. Further, smaller sized stalactites dissolved comparatively faster than the larger ones which is confirmed with the complete dissolution of smallest stalactite (marked by blue arrow head in fig. 2a) and partial dissolution of larger ones (red arrow heads in fig. $2 a$ ) in fig. $2 \mathrm{~b}$. The dissolution seems to start at random location (with the condition that these locations must be in direct contact with substrate) on individual stalactites. While the dissolution occurred from the locations directly in contact with the substrate, growth seems to follow the opposite trend. This means growth was only observed in locations on the stalactites which are not in direct contact with the substrate. For example, area marked by green arrow head in fig. 2 a not in direct contact with GC substrate started to grow at the same time (see fig. 2b). In addition, few individual structures (marked by black colored lollypop in fig. 2a) on top of stalactite also coalesced together to form larger structure (see black color lollypop mark in fig. 2b). 

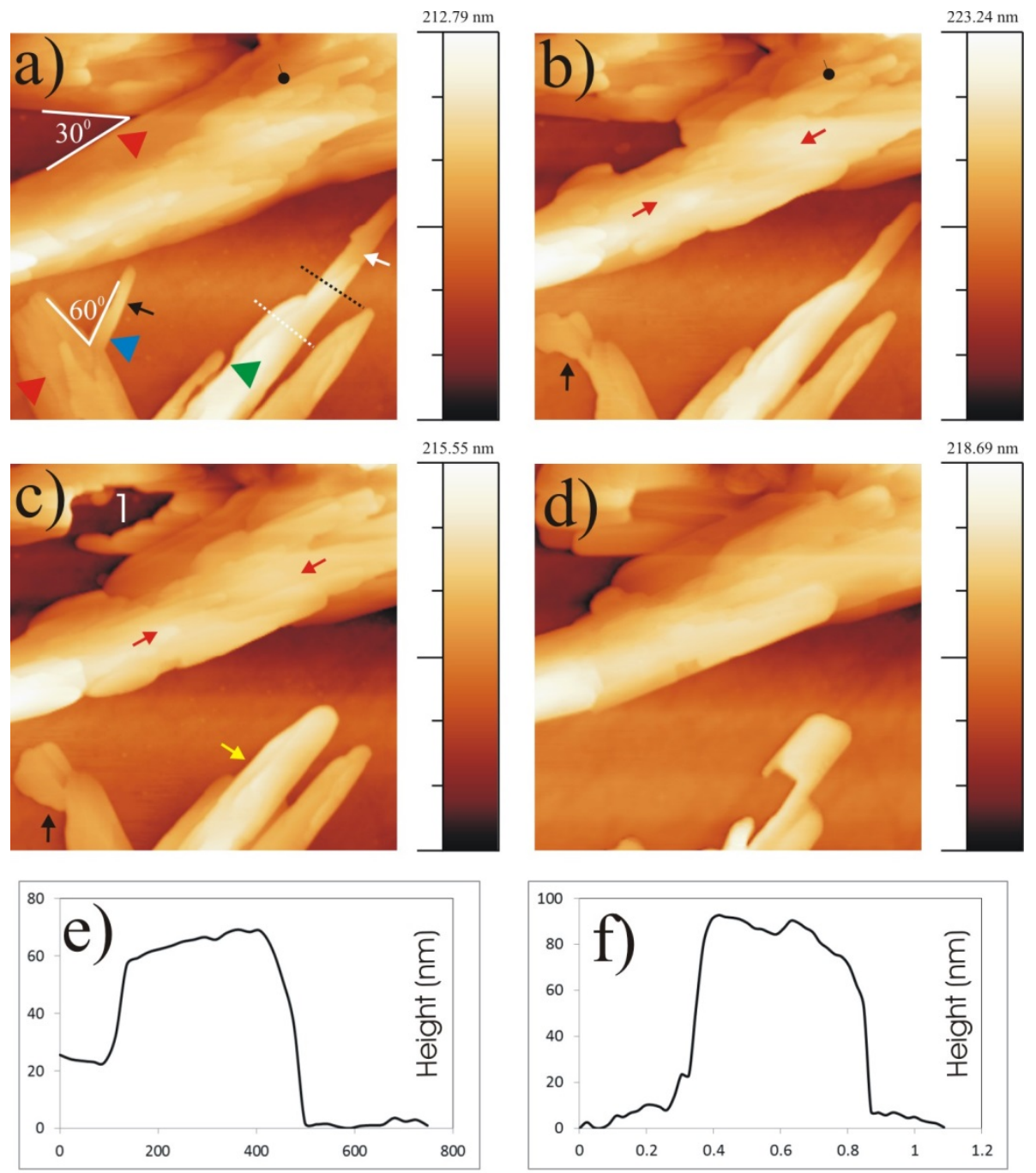

Width $(\mathrm{nm})$

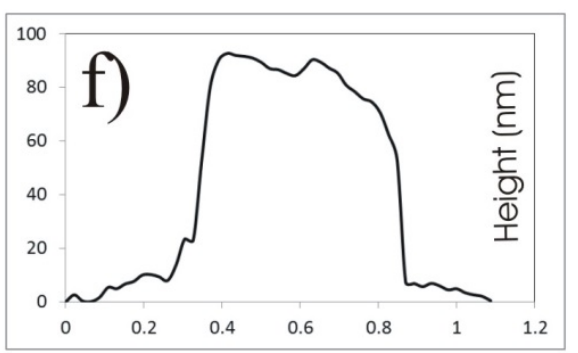

Width $(\mu \mathrm{m})$

Figure 2. Morphological changes observed by tapping mode AFM at different time intervals for LG crystals after recrystallizing $0.4 \mathrm{mg} / \mathrm{mL} \mathrm{LG} \mathrm{solution}$ (chloroform solvent) on to glassy carbon (GC) substrate. Figure 2a taken after $\sim 13$ min shows stalactite-like-structures of variable lengths, widths and heights. Morphological changes observed at $\sim 70 \mathrm{~min}$ and $\sim 257 \mathrm{~min}$ are shown in fig. $2 \mathrm{~b}$ and $2 \mathrm{c}$ respectively. Figure $2 \mathrm{~d}$, taken at $\sim 1276 \mathrm{~min}$ demonstrated the saturation morphology. Details are explained in text under heading "LG crystallization on GC" section. Figure $2 \mathrm{e}$ and $2 \mathrm{f}$ show the height profiles across the black and white dotted line in fig. 2 a.

The growth/dissolution of some of the stalactites continued even after 4 hours (fig. 2 c, 257 min). In addition, a stalactite which was partially dissolved previously (see fig. $2 \mathrm{~b}$, black arrow) started to grow (see fig. $2 \mathrm{c}$, black arrow). It is interesting to note that the dynamics of dissolution/ growth of regions of stalactites in direct contact with or located very close to the GC substrates is quite prominent than the areas which are far away from GC substrate. See for example, the top surface of the largest stalactite (marked by red arrows in fig. 2b) has rarely undergone any visible morphological change even after scanning more than 4 hours (red arrows, fig. $2 \mathrm{c}$ ). The recrystallization process became saturated at $\sim 21$ hours (fig. 2d, $1276 \mathrm{~min}$ ) and no further change was observed on the surface even though scanning was performed for up to 24 hours. At this stage, few of those regions which was either dissolved or grown previously was found to have followed the opposite trend i.e., they have overlapped one each other to form a complete film (marked number ' 1 ' in fig. $2 \mathrm{c}$ completely overlapped in fig. $2 \mathrm{~d}$ ) or completely/partially dissolved again (black/yellow arrow marked areas in fig. 2c dissolved completely or partially in fig. 2d). The morphological changes on a GC substrate seems to be not so significant compared to the large morphological changes occurred on HOPG at the same time period. Also the saturation time period observed on GC was found to be considerably higher than on HOPG where surface saturation was reached at $\sim 18$ hours for $0.4 \mathrm{mg} / \mathrm{mL}$ LG solution.

\subsection{LG Recrystallization on Glass}

Unlike GC, on polar, amorphous substrates like glass, recrystallization of $L G$ from chloroform resulted in thin film formation (see fig. 3a, $\sim 14 \mathrm{~min}$ ). At the initial stage (fig. 3a), most of the glass surface was found to be covered with thin 
films with occasional pits developed in some areas (few pits are marked by black arrows in fig. 3a). A closer look also revealed the thin films are formed in a layer-by-layer fashion (see the white arrows in fig. 3a). The height measured across one such visible layer (white dotted line in fig. 3a) with respect to layer underneath gives a height of $\sim 3.1 \mathrm{~nm}$ (see fig. $3 \mathrm{e}$ height profile). Layers visible on other areas of the image
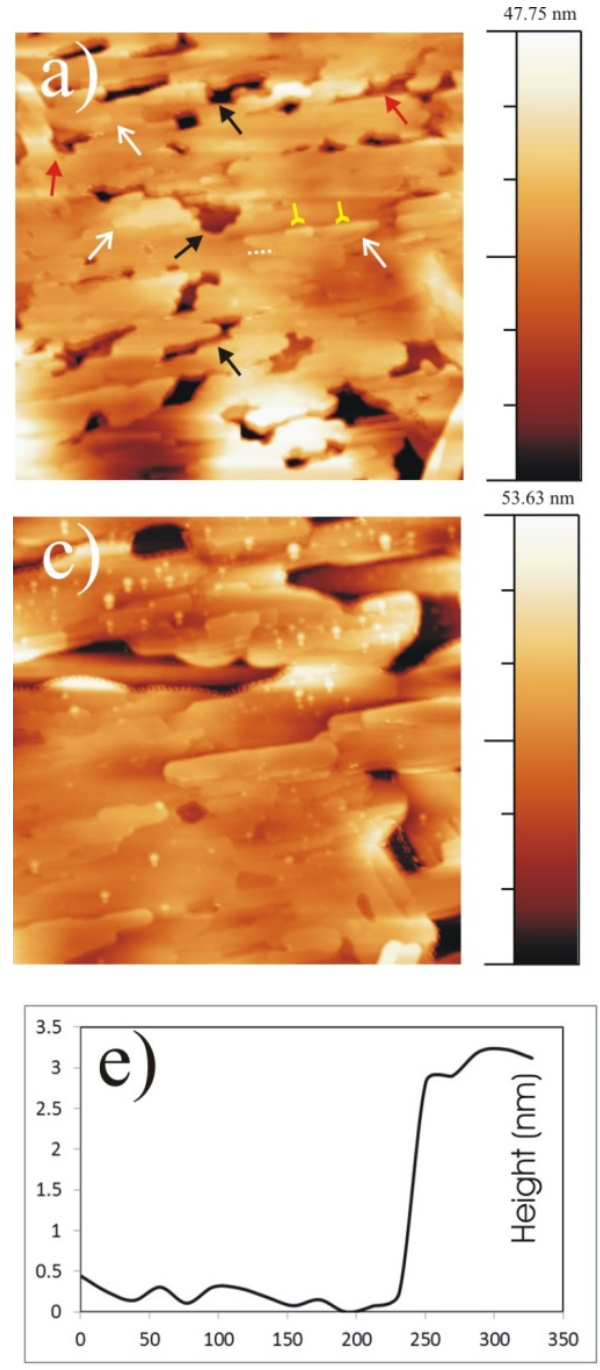

Width (nm) also gave similar heights and hence not discussed further. Another interesting observation is the gradual decrease in average size of the layers deposited at LG-glass interface from bottom (LG-glass interface) towards top (close to the reader). For instance the areas marked by bifurcated yellow arrows in fig. 3a shows the gradual decrease in the island sizes from bottom towards top.
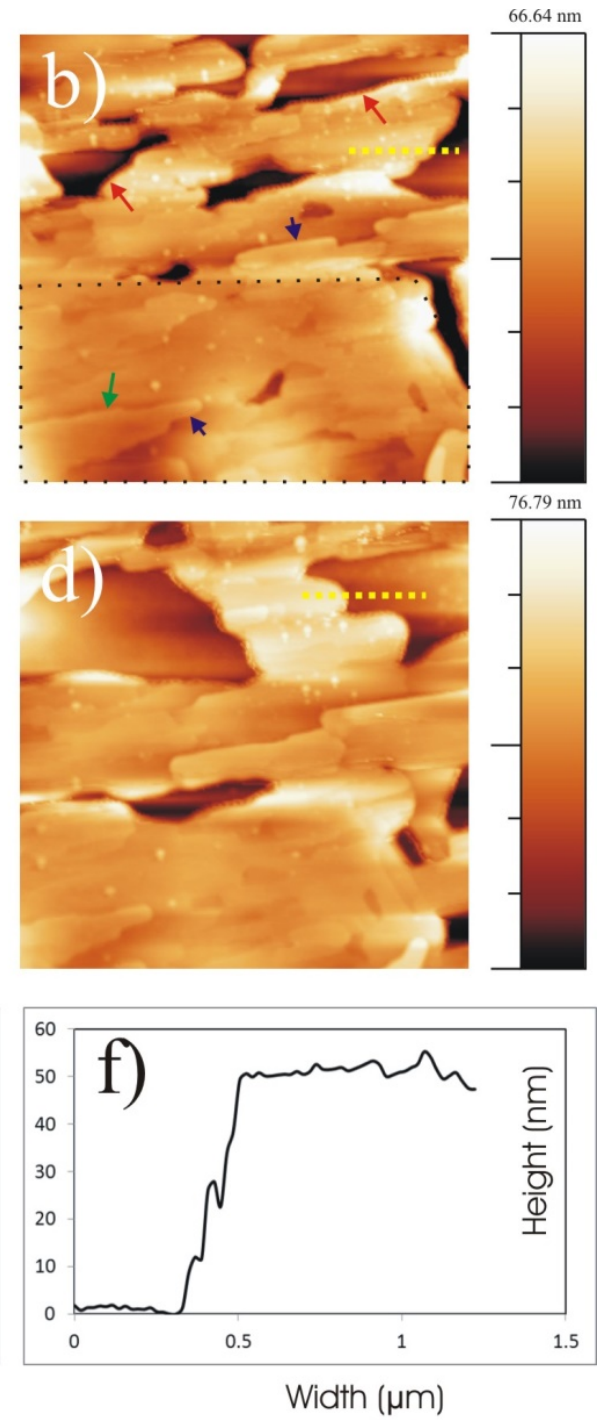

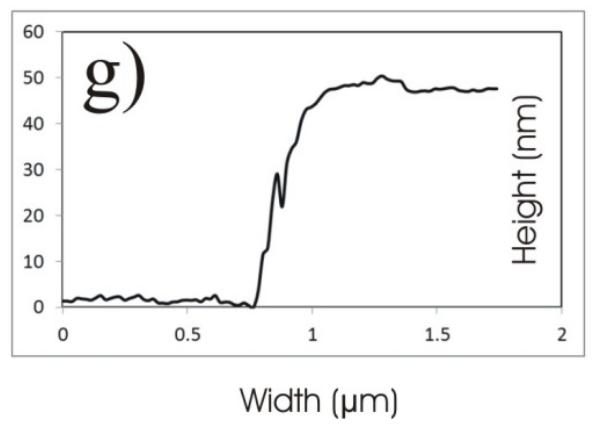

Figure 3. AFM images at different time intervals showing the topographical changes of LG crystals after recrystallizing $0.4 \mathrm{mg} / \mathrm{mL}$ LG solution (chloroform solvent) on to glass substrate. Figure 3a taken after $\sim 14$ min shows thin films with many pits at the LG-glass interface. Morphological changes at different time intervals: fig. 3b, c, d taken after $\sim 132, \sim 668$ and $\sim 1145$ min demonstrates the recrystallization behavior (for details see text under section 'LG recrystallization on glass'). Figure $3 \mathrm{e}, \mathrm{f}$ and $\mathrm{g}$ demonstrates height profiles across the white and yellow dotted lines in fig. $3 \mathrm{a}, \mathrm{b}$ and $\mathrm{d}$ respectively. 
Thin films observed during the initial stages in fig. 3a started to coalesce together to form larger islands after $\sim 2$ hours (fig. $3 \mathrm{~b}, \sim 132 \mathrm{~min}$ ). For example, the square area marked by black dots in fig. $3 \mathrm{~b}$ shows the disappearance of pits previously observed at the exact location in fig. $3 \mathrm{a}$ to form an almost complete film with very few distinguishable pits. At the same time area lying outside the black dots in fig. $3 \mathrm{~b}$ resulted in dissolution/merging of some of the islands. As a consequence, small pits observed for that area (red arrows, fig. 3a) developed in to larger pits (red arrows, fig. 3b). Further, it also found that the films have grown in a layer-by-layer fashion following Frank-Van der Merwe mechanism [17]. As an example, see grown layer marked by green arrow in fig. $3 \mathrm{~b}$ where the top layer has grown over the already formed layer underneath. The layers which have grown over the other layers seem to have structures similar to those observed for HOPG (see navy blue marked arrows in fig. 3b). On the other hand, those formed at the LG-glass interface do not have such features. Few rounded globules appeared on the surface during the imaging (see the particles in fig. 3b) is believed to be dust particles from the environment which got deposited during long scanning hours.

Dissolution and growth process continued even after waiting up to $\sim 11$ hours (fig. $3 \mathrm{c}, \sim 668 \mathrm{~min}$ ). As a consequence few of the pits developed in to larger pits whereas few others became tiny due to the coalescence of adjacent thin film. Looking at the relatively minute morphological changes that have occurred between fig. $3 \mathrm{~b}$ and fig. $3 \mathrm{c}$ taken at a time gap of $\sim 9$ hours, it is safe to assume that the recrystallization process has slowed down considerably. This is in stark contrast to the relatively fast morphological change occurred within first two hours (see fig. 3a and fig. 3b). This observed trend in growth and dissolution behavior is similar to those observed on $\mathrm{GC}$ and HOPG substrate. Although imaging of that surface location was continued for up to $\sim 22$ hours, no visual change in surface morphology was observed after $\sim 19$ hours (fig. 3d, $\sim 1145 \mathrm{~min}$ ) indicating the growth/dissolution process has reached a saturation point. This saturation time period is confirmed by scanning another area of freshly recrystallized LG on glass. The heights of thin film in direct contact with the glass surface (assuming the lowest visible surface to be glass) were measured at the same location for fig. $3 \mathrm{~b}, \mathrm{~d}$ (yellow dotted line) taken at a time gap of $\sim 2$ and 19 hours and found to remain constant $(\sim 50 \mathrm{~nm})$ during recrystallization (see fig. $3 \mathrm{f}, 3 \mathrm{~g}$ ). Height at the same location in fig 3 a could not be measured as the lowest surface was not visible. By comparing LG recrystallization on glass with that on HOPG and $\mathrm{GC}$, it is found that the recrystallization saturation time lies between HOPG and GC. It is important to recall, the saturation time of $0.4 \mathrm{mg} / \mathrm{mL} \mathrm{LG}$ crystallization on HOPG and GC is $\sim 18$ hours and 21 hours respectively.

\subsection{LG Recrystallization on Mica}

Topographical changes at different time intervals after recrystallizing $0.4 \mathrm{mg} / \mathrm{mL} \mathrm{LG}$ mass on to mica substrate are shown in fig. 4. Recrystallization of LG on mica followed an entirely different behavior compared to those discussed for other substrates in the above paragraphs. Instead of any parallelograms, stalactites or large thin films, only small islands of irregular shape and size are observed. Fig. 4a taken after $\sim 14$ min. demonstrates one such area of mica surface after recrystallizing $0.4 \mathrm{mg} / \mathrm{mL} \mathrm{LG}$ mass on to it. Similar islands are also observed in other experiments on different areas of mica indicating that they are homogeneously distributed over the mica surface. The average heights of these islands were found to be between $\sim 1-5 \mathrm{~nm}$. As an example, see the height profile along black dotted line in fig. 4a giving heights between 1-5 nm (black profile in fig. 4e). Interestingly, these small islands begin to swell as well as newer islands started to emerge on the mica surface only after $\sim 42$ minutes (see fig. $4 \mathrm{~b}$ ). As time passed further, some of these small islands started to merge together to form larger islands by the diffusion of LG molecules (fig. 4c, 126 min). For example, area marked by white dotted line in fig. $4 \mathrm{~b}$ combined to form a larger island (see white dotted marked area in fig. 4c). In addition, these individual islands were found to be composed of multilayers (see white arrows in fig. 4c). Height profile across the black line in fig. 4c demonstrates $\sim 2 \mathrm{~nm}$ height for the layer (fig. $4 \mathrm{f}$ ). The height of bottom visible layer in direct contact with the substrate (assuming it to be mica surface) in fig. $4 \mathrm{c}$ was also found to be $\sim 2 \mathrm{~nm}$ (profile not shown). A closer look also revealed that merging of the individual islands occurred in a layer by layer fashion with the bottom layers coalesce first followed by the top layers. Although the number of islands and their sizes started to increase until this point but their heights remain constant. This is confirmed by measuring the heights along the same line (see colored dotted lines in fig. $4 \mathrm{a}, \mathrm{b}$ and c). Color of the dotted lines in figures corresponds to the exact colors shown in fig. 4e profile. Recrystallization has reached a saturation period at $\sim 19$ hours (see fig. $4 \mathrm{~d}, \sim 1108$ min) and no further morphological change occurred after this time period. This saturation time period is slightly lower compared GC and glass but higher than that on HOPG substrate. Comparing fig. $4 \mathrm{c}$ and $4 \mathrm{~d}$ which was recorded within a time gap of $\sim 15$ hours, smaller islands further coalesce together to form large ones. At this stage, heights of the islands were found to have several orders higher (see the red dotted line in fig. $4 \mathrm{~d}$ and red profile in fig. 4e). This observation holds true for the entire imaging area shown. 

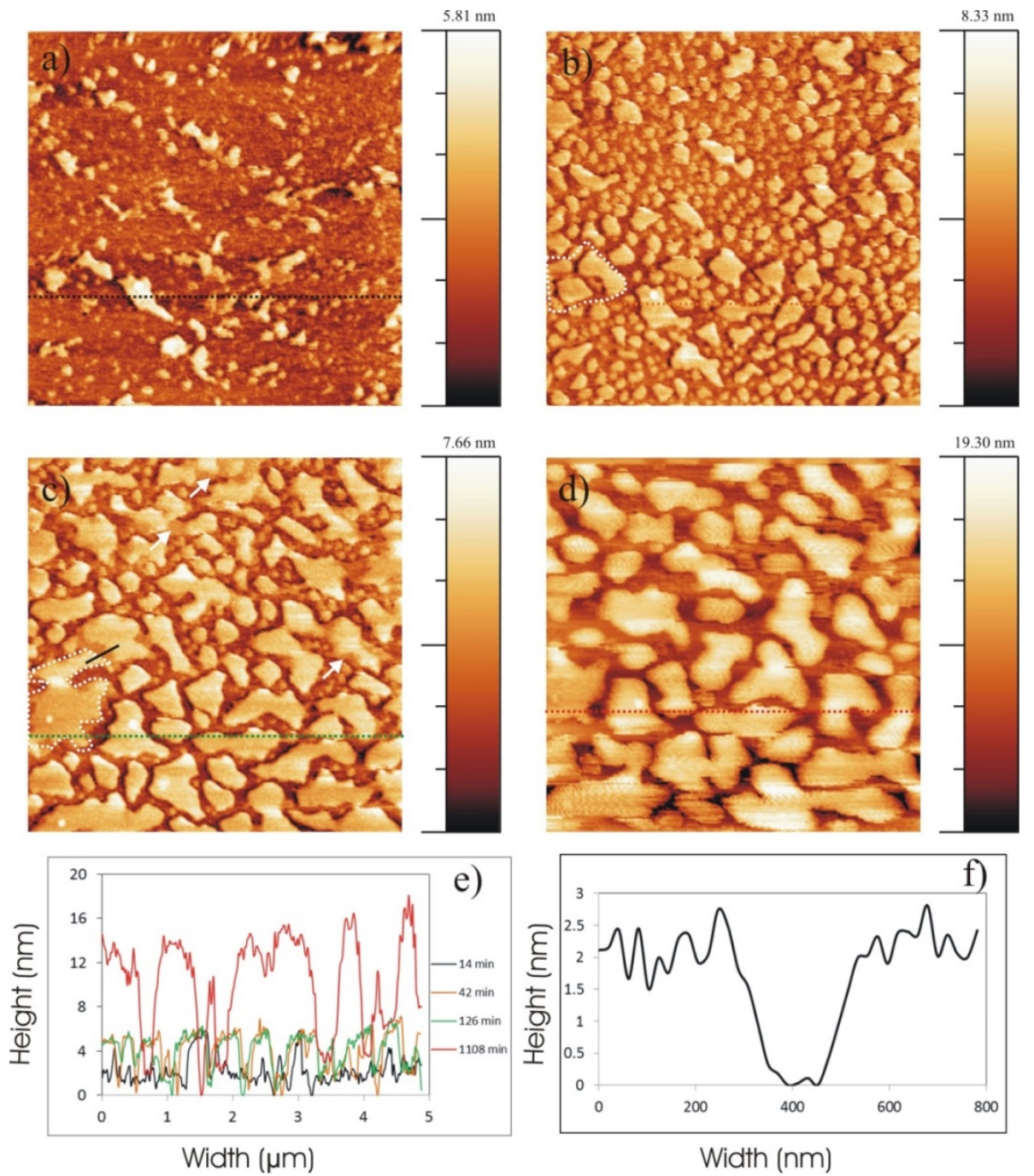

Figure 4. AFM images at different time intervals showing the morphological changes of LG mass after recrystallizing $0.4 \mathrm{mg} / \mathrm{mL} \mathrm{LG} \mathrm{solution} \mathrm{(chloroform}$ solvent) on to mica substrate. Figure $4 \mathrm{a}$ taken after $\sim 14$ min shows homogeneously distributed islands of different shapes and sizes. Figure $4 \mathrm{~b}, \mathrm{c}$ taken after $\sim 42 \mathrm{~min}$ and $\sim 126 \mathrm{~min}$ demonstrates merging of several small island to grow in to bigger ones. At $\sim 1108 \mathrm{~min}$, island growth has completely ceased (fig. $4 \mathrm{~d}$ ) and no further change was observed. Figure $4 \mathrm{e}$ shows the height profiles along the dotted lines in all the images (dotted colored lines in figures corresponds to the same color profile in fig. $4 \mathrm{e})$. Figure $4 \mathrm{f}$ represents height profile along the black line in fig. $4 \mathrm{c}$.

\section{Discussion}

AFM imaging at different time intervals demonstrated for the first time the substrate effect on the growth and orientation of LG molecules. The morphological variations of LG molecule is presented in the table below.

\begin{tabular}{|c|c|c|}
\hline Substrates & Nonpolar & Polar \\
\hline Crystalline & $\begin{array}{c}\text { HOPG } \\
\text { (Parallelogram-like-structures) }\end{array}$ & $\begin{array}{c}\text { Mica } \\
\text { (Small islands of } \\
\text { irregular shape and } \\
\text { size) }\end{array}$ \\
\hline Amorphous & $\begin{array}{c}\mathrm{GC} \\
\text { (Stalactite-like-structures) }\end{array}$ & $\begin{array}{c}\text { Glass } \\
(\text { Thin film })\end{array}$ \\
\hline
\end{tabular}

Individual parallelogram-like-structures observed on HOPG is attributed to its nonpolar, crystalline nature. LG molecules have linear carbon-carbon (C-C) chains and their $\mathrm{C}-\mathrm{C}$ chain length matches exactly to that of the $\mathrm{C}-\mathrm{C}$ bond length of HOPG. This well matching $\mathrm{C}-\mathrm{C}$ bond length provided the driving force for the epitaxial growth of $L G$ molecules in an ordered manner at the initial stages. The term epitaxy refers to the growth of crystalline overlayer on a crystalline substrate. Epitaxial growth on HOPG resulting in the formation of ordered structures is well documented [18, 19]. In fact hetero-epitaxial growth of long chain alkanes producing similar kind of structures is already reported in literature [18]. The distinct widths varying within $\sim 100-400$ $\mathrm{nm}$ must be due to their inherent properties of the molecular architecture which provides lowest energy for such orientations. The homogeneous distribution of these structures on HOPG can be attributed to the atomically flat nature of HOPG providing the lowest activation energy on which LG molecules can diffuse with relative ease. This is further aided by the fact that the interaction between LG (polar) and HOPG (nonpolar) is comparatively weaker due to their difference in polarity. Arrangement of parallelogram-like-structures in triangular fashion reflects 
the hexagonal symmetry of HOPG substrate [20]. The average height of a single visible layer was found to be $\sim 3.1$ $\mathrm{nm}$. Considering the length of a single LG molecule to be $1.8 \mathrm{~nm}$ (chain length of dodecane is $1.8 \mathrm{~nm}$ [21]), such a layer should be formed by the close packing of two vertically standing LG molecule one over the other. But such an arrangement should give a height of $3.6 \mathrm{~nm}$ against $3.1 \mathrm{~nm}$ observed in this case. This may be attributed to the surface deformation by the applied force during the AFM measurement. Deformations observed on soft materials by AFM probe force is well documented [22]. With passage of time, some of these structures have grown while others dissolved simultaneously and as seen in figure 1, the topographical changes slowed down with time. It is believed that the growth and dissolution of these structures are solvent (chloroform) driven. In the initial stages of recrystallization, when LG solution was deposited on to the HOPG surface, more number of solvent molecules was present in the deposited crystal. These higher numbers of solvent molecules were responsible for the faster diffusion at the initial stage. As time passed by, although most of the solvent molecules evaporated, it may happen that some of these solvent molecules are trapped within the LG molecules and their evaporation took much longer. Hence diffusion has slowed down considerably due to the presence of lesser amount of solvent. The surface saturation has reached when all the solvents are evaporated and no further changes are observed $\sim 18$ hours. This solution driven recrystallization mechanism holds true for all other substrates discussed from here onwards and hence will not be discussed further. During the growth of these structures their vertical height remained constant. In other words growth was observed only along their length and width. This makes sense as adding LG mass below the area of the parallelogram-like-structure in direct contact with HOPG or above it requires a considerable amount of activation energy and hence is not energetically favorable. During growth their length increased at a faster rate compared to their width. This is due to the larger available area across their width compared to the smaller area at the growth end along the length. Assuming similar number of LG molecules gets arrived at the nucleation site at a particular time interval, these molecules must distribute themselves equally to the larger available area across width compared to the small area at the growth end along length. Hence their length increased at a faster rate compared to their width. What happened to the structures which disappeared with time? The flash point of LG molecules is $\sim 134.3^{\circ} \mathrm{C}$ and the experiments were performed at room temperature $\left(20-24^{0} \mathrm{C}\right)$ completely ruling out their evaporation from the HOPG surface. The only probable explanation would be their absorption on to the HOPG surface to form thin film. Although no self-assembly study of LG on HOPG is done, however a self-assembly study of its homologue 1, 12 dodecanediol demonstrated that the carbon chain lies parallel to the HOPG surface [23]. This is a strong indication that the parallelograms dissolved to form a thin film. If polar-polar interaction between LG molecules is stronger then what drives the dissolution process of these structures? The argument would be to lower surface energy, the vertical standing LG molecules (less stable structure) undergo surface rearrangement to form a thin film where the carbon-carbon chain lies in parallel (more stable structure) orientation with respect to HOPG surface. The well matching carbon-carbon (C-C) bond distance between LG and HOPG provides a favorable environment for this rearrangement resulting in a hetero-epitaxial growth. The above explained mechanism is also responsible for the formation of similar structures for long chain alkanes e.g., dotriacontane $\left(\mathrm{C}_{32} \mathrm{H}_{66}\right)$ and tritriacontane $\left(\mathrm{C}_{33} \mathrm{H}_{68}\right)$ on HOPG demonstrated by Leunissen et al. [18]. If dissolution is favorable to give stability to the arrangement then why few parallelogram structures also grow simultaneously? One hypothesis would be the saturation of thin film in the nearby regions of grown parallelogram. As a result of this the molecules nearby its capture zone have nowhere to go but to get added to the existing parallelogram and hence the growth has occurred. The existence of parallelogram-like-structures and thin film together indicates the recrystallization process switched from Volmer-Weber mode to Stranski-Krastanov mode [17].

Even though GC is nonpolar, however the surface roughness which is higher than $100 \mathrm{~nm}$ (see fig. 3 in citation [24]) probably played a major role hindering the smooth diffusion of LG mass resulting in the formation of in-homogeneously distributed stalactite structures on the surface. The irregular shape of these stalactites in the initial stage of recrystallization is probably due to the amorphous nature of GC. In the absence of any regular arrangement on GC (amorphous), the incoming LG molecules are oriented randomly resulting in a non-epitaxial growth. The term non-epitaxial growth refers to the random distribution of molecules on a substrate. In addition, being amorphous one would expect the crystallized structures not to follow any specific direction. However, certain directional nature of few stalactites (see fig. 2a with $30^{\circ}$ and $60^{\circ}$ angle) although unlike those on HOPG is slightly surprising. Commercially available GC has many scratches and these scratches are oriented in different directions (see citation [24] fig. 3). One would expect that these scratches may act as the preferential nucleation sites for the incoming LG molecules from the solution and provide certain directions for the LG molecule growth. The directional nature of stalactite shown in fig. $2 \mathrm{a}$ most probably developed due to the above mentioned reason. Why do they grow as stalactites on GC? It is believed that non-polarity plays an important role in stalactite formation. To explain it further, GC being nonpolar, the nonpolar end of incoming LG molecules at the closest vicinity of GC will get attached to it first. The second batch of molecules will then have to get attached to the polar free end of the already attached molecules on GC as polar-polar interaction is stronger. This will make the top surface of deposited LG molecule also non polar and hence the next batch of LG molecules will have the preference to get deposited either on 
GC or on the already formed LG layer. Ultimately some LG molecules will get attached to GC substrate and other on top of already formed LG layer. This alternating deposition will continue until all the molecules from solution get deposited. However, once the first layer is formed, the number of molecules in droplet decreased further and hence less number of molecules remains in the droplet. This stepwise decrease in number of recrystallized molecule as well as their preferential absorption until complete recrystallization resulted in the formation of stalactites. The dissolution has also occurred at the interface between LG and GC. Similar factor as explained for HOPG most probably drives the dissolution in case of GC also. Transition to thin film in some areas along with the existence of stalactites most probably indicates a shifting of nucleation mechanism from pure Volmer-Weber type towards Stranski-Krastanov type [17].

Interestingly on polar amorphous substrate like glass the imaged area was found to be almost completely covered by the thin film. In the initial stage of recrystallization, the driving force for thin film formation is believed to be a combined effect of both LG-glass and LG-LG (inter molecular hydrogen bonding between $-\mathrm{OH}$ groups within LG molecules) polar interaction. Since glass substrate and LG molecules both are polar in nature, so the incoming molecules had the luxury of either depositing on glass or interact with the other LG molecules. It is probably this combined effect where one polar group (-OH group) of LG binds to the substrate and other one binds to the - $\mathrm{OH}$ group of adjacently placed LG to form the complete film. In other words, the recrystallization followed the well-known Frank-van der Merwe mechanism [17]. Once the film is formed, rearrangement of film started to occur to minimize the interfacial energy. The consequence is dissolution and rearrangement of few areas on these films at the latter stages of crystallization. The morphological changes appeared here is completely different to those observed on HOPG or GC indicating that substrate polarity plays an important role in determining the recrystallization behavior.

Finally on mica which is polar and crystalline, patches of small islands of irregular shape and size are observed which I attribute to the stronger LG-mica interaction than LG-LG (inter molecular hydrogen bonding between - $\mathrm{OH}$ groups within LG molecules) interaction. This LG-mica interaction resulted in LG molecules from solution phase to get attached to mica preferably forming a large number of small irregular shaped islands. The reason for the formation of such small patches at the initial stage is attributed to stronger LG-Mica polar-polar interaction. Mica being crystalline and negatively charged, its interaction with incoming LG molecules is much stronger compared to the intermolecular polar interaction between LG molecules. Hence, the incoming LG molecules from solution phase got attached to the mica surface preferentially forming large number of islands. These islands formed on the mica substrate in the initial stages of crystallization are completely different from those grown on glass. Just to recall thin films observed on a glass substrate still have some regular shapes e.g. formed by close packing of individual parallelogram like structures. Growth of these small islands and their coarsening followed Frank-van der Merwe growth mechanism [17] to develop in to larger islands at the latter stages. An average height of $\sim 1-5 \mathrm{~nm}$ of these islands in the initial stage of recrystallization (up to 2 hours) was observed indicating a random orientation of LG molecules on mica. Further no change in heights of these structures in the first couple of hours (see height profiles for in fig. 4e) combined with the island growth strongly supports that the molecular diffusion was strictly two dimensional e.g. along $\mathrm{X}$ and $\mathrm{Y}$ axes. Layer heights increase up to $\sim 3-4$ times in the final stage is indicative of layer by layer growth of LG thin film following a Frank-van der Merwe type of nucleation behavior. The complete merging of individual islands probably didn't occur due to the unavailability of enough LG molecules as a low concentration of $0.4 \mathrm{mg} / \mathrm{mL}$ LG solution was used.

The recrystallization saturation time period differed on different substrates used. The time period followed the following trend

$$
\text { Glassy carbon }>\text { glass }>\text { mica }>\text { HOPG }
$$

This difference can be explained based on their roughness and polarity. Commercial GC substrates have higher roughness ( 100 nm [23]) compared to all other substrates used. This higher roughness will provide hindrance to the molecular diffusion thus requiring higher energy for the movement of LG molecules. On the other hand, glass being much smoother, the diffusion barrier is lower than that of GC. For mica and HOPG though both are atomically flat and thus diffusion barrier is low, however mica being polar, the stronger LG-Mica interaction will prevent LG molecular diffusion to a greater extent than HOPG which is nonpolar in nature and provides a weaker interaction with LG molecules.

\section{Conclusions}

This study is a first step towards understanding at nano level how substrate properties affect the recrystallization of LG molecules which is used as a skin conditioner in many skin cosmetic products. The results reveal that both surface polarity and roughness affects the recrystallization process on all investigated substrates. On polar substrates like mica and glass, strong polar-polar interaction between substrate and LG molecules was the driving force for thin film formation which followed Frank-Van der Merwe nucleation mechanism. On the other hand on nonpolar substrate like GC, existence of stalactites and thin films at the final stage of recrystallization indicated a transition from Volmer-Weber in to a Stranski-Krastanov nucleation mechanism. HOPG behaved as a special material due to its non-polarity, crystalline nature where exact $\mathrm{c}-\mathrm{c}$ bond distance matching with LG resulted in the formation of parallelogram-like-structures. However, LG crystallization on HOPG also followed a transition from Volmer-Weber in 
to a Stranski-Krastanov nucleation mechanism. The molecular diffusion leading to growth and dissolution was also found to be controlled by both roughness and polarity of the investigated surfaces. It is believed the detailed understanding of their growth/dissolution mechanism as well as how substrate properties affect them is very interesting for scientists working in cosmetic industries and will behave as a catalyst to study many other constituents used in cosmetics. Better understanding of these processes at the nano-level will ultimately work as a stepping stone to prepare high quality cosmetic products in the near future.

\section{REFERENCES}

[1] R. Pillai, G. Schmaus, A. Pfeiffer, S. Lange, A. Trunet, 1,2-Alkanediols for Cosmetic Preservation, Cosmetics and Toiletries, Vol. 123, Issue 10, 53-64, 2008.

[2] H. J. Sagitani, Formation of $\mathrm{O} / \mathrm{W}$ emulsions by surfactant phase emulsification and the solution behavior of nonionic surfactant system in the emulsification process, Dispersion Sci. Technol., Vol. 9, Issue 2, 115-129, 1988.

[3] S. J. Paynter, L. O’Neil, B. J. Fuller, R. W. Shaw, Membrane permeability of human oocytes in the presence of the cryoprotectantpropane-1,2-diol, Fertility Sterility, Vol. 75, No. 3, 532-538, 2001.

[4] A. E. Sagiv, S. Disktein, A. Ingeber, The efficiency of humectants as skin moisturizers in the presence of oil, Skin Res. Technol., Vol. 7, 32-35, 2001.

[5] S. B. Levy, A. M. Dulichan, M. Helman, Safety of a preservative system containing 1,2-hexanediol and caprylyl glycol, Cutaneous and Ocular Toxicology, Vol. 28(1), 23-24, 2009.

[6] W. Johnson Jr, W.F. Bergfeld, D.V. Belsito, R.A. Hill, C.D. Klaassen, D. Liebler, J.G. Marks Jr., R.C. Shank, T.J. Slaga, P.W. Snuder, F.A. Andersen, Safety Assessment of 1,2-Glycols as Used in Cosmetics, International journal of toxicology, Vol. 31 (supplement 2), 147s-168S, 2012.

[7] Júnior CO, le Hyaric M, Costa CF, Corrêa TA, Taveira AF, Araújo DP, Reis EF, Lourenço MC, Vicente FR, Almeida MV., Preparation and antitubercular activity of lipophilic diamines and amino alcohols, Mem Inst Oswaldo Cruz., Vol. 104 (5), 703-5, Aug 2009.

[8] Naidek KP, Bianconi F, da Rocha TC, Zanchet D, Bonacin JA, Novak MA, Vaz Md, Winnischofer H., Structure and morphology of spinel MFe2O4 (M=Fe, Co, Ni) nanoparticles chemically synthesized from heterometallic complexes, J Colloid Interface Sci., Vol. 358, Issue 1, 39-46, 2011.

[9] F. A. Freitas, D. Licursi, E. R. Lachter, A. M. R. Galletti, C. Antonetti, T. C. Brito, R. S. V. Nascimento, Heterogeneous catalysis for the ketalisation of ethyl levulinate with 1,2-dodecanediol: Opening the way to a new class of bio-degradable surfactants, Catalysis Communications, Vol. 73, 84-87, 2016.

[10] Greg Haugstad, Atomic Force Microscopy: Understanding Basic Modes and Advanced Applications, Wiley (September 2012).
[11] Ardeshir Danesh, Simon D. Connell, Martyn C. Davies, Clive J. Roberts, Saul J. B. Tendler, Phillip M. Williams, M. J. Wilkins, An In Situ Dissolution Study of Aspirin Crystal Planes (100) and (001) by Atomic Force Microscopy, Pharmaceutical Research, Vol. 18, No. 3, 299-303, 2001.

[12] Tonglei Li, Kenneth R. Morris, Kinam Park, Influence of Solvent and Crystalline Supramolecular Structure on the Formation of Etching Patterns on Acetaminophen Single Crystals: A Study with Atomic Force Microscopy and Computer Simulation, J. Phys. Chem. B, Vol. 104, No. 9, 2019-2032, 2000.

[13] Drug Dissolution Studies with Atomic Force Microscopy, Bruker Application note, 2010.

[14] L. Itzel Meza, Michael W. Anderson, Ben Slater, Jonathan R. Aggera, In situ atomic force microscopy of zeolite A dissolution, Phys. Chem. Chem. Phys., Vol. 10, 5066-5076, 2008.

[15] Justyna Pers, Bogdan Barwinski, Milosz Grodzicki, Antoni Ciszewski, AFM studies of pits formation on $\mathrm{KBr}(100)$ during its dissolution by water, Materials Science-Poland, Vol. 34(4), 863-867, 2016.

[16] I. Horcas, R. Fernandez, J.M. Gomez-Rodriguez, J. Colchero, J. Gomez-Herrero and A. M. Baro, WSXM: A software for scanning probe microscopy and a tool for nanotechnology, Rev. Sci. Instrum. Vol. 78, 013705, 2007.

[17] Ivan V. Markov, Crystal growth for beginners: Fundamentals of nucleation, crystal growth and epitaxy (2nd edition), World Scientific Publishing Company, February 2004.

[18] Mirjam E. Leunissen, W. Sander Graswinckel, Willem J. P. van Enckevort, and Elias Vlieg, Epitaxial Nucleation and Growth of n-Alkane Crystals on Graphite (0001), Crystal Growth \& Design, Vol. 4, No. 2, 361-367, 2004.

[19] Arrigoni Claire, Schull Guillaume, Bléger David, Douillard Ludovic, Fiorini-Debuisschert Céline, Mathevet Fabrice, Kreher David, Attias André-Jean and Charra Fabrice, Structure and Epitaxial Registry on Graphite of a Series of Nanoporous Self-Assembled Molecular Monolayers, J. Phys. Chem. Lett., Vol. 1 (1), 190-194, 2010.

[20] Chong-yang Liu, Hsiangpin Chang, and Allen J. Bard, Large scale hexagonal domain like structures superimposed on the atomic corrugation of a graphite surface observed by scanning tunneling microscopy, Langmuir, Vol. 7, 1138-1142, 1991.

[21] J. R. Lu, R. K. Thomas, B. P. Binks, P. D.I Fletcher, J, Penfold, Structure and Composition of Dodecane Layers Spread on Aqueous Solutions of Dodecyl- and Hexadecyltrimethylamm onium Bromides Studied by Neutron Reflection, J. Phys. Chem., Vol. 99, No. 12, 4113-4213, 1995.

[22] Albrecht L Weisenhorn, Mitra Khorsandi, Sandor Kasas, Vassilis Gotzos, Hans-Jurgen Butt, Deformation and height anomaly of soft surfaces studied with an AFM, Nanotechnology, Vol. 4, 106-113, 1993.

[23] C. L. Claypool, F. Faglioni, W. A. Goddard III, H. B. Gray, N. S. Lewis, R. A. Marcus, Source of Image Contrast in STM Images of Functionalized Alkanes on Graphite: A Systematic Functional Group Approach, J., Phys. Chem. B, Vol. 101, 5978-5995, 1997.

[24] Gregory J. Barbante, Conor F. Hogan, Adam Mechler and Andrew B. Hughes, Electrochemiluminescence of surface bound microparticles of ruthenium complexes J. Mater. Chem., Vol. 20, 891-899, 2010. 